\title{
Star Formation in Nuclear Rings of Barred-Spiral Galaxies
}

\author{
Woong-Tae Kim and Woo-Young Seo \\ CEOU, Astronomy Program, Department of Physics \& Astronomy, \\ Seoul National University, Seoul 151-742, Republic of Korea \\ email: wkim@astro.snu.ac.kr, seowy@astro.snu.ac.kr
}

\begin{abstract}
We study star formation occurring in nuclear rings of barred-spiral galaxies by using hydrodynamic simulations with the prescriptions of star formation and feedback included. In models without spiral arms, the star formation rate (SFR) in a ring exhibits a strong primary burst at early time and declines to small values at late time. The early burst is caused by a rapid gas infall due to the bar growth, consuming most of the gas inside the bar regions. On the other hand, models with spiral arms show multiple starburst activities at late time caused by arm-induced gas inflows, provided that the arm pattern speed is slower than that of the bar. The SFR in models with spirals is larger by a factor of $\sim 1.4-4.0$ than that in the bar-only models, with larger values corresponding to stronger and slower arms. In all models, young star clusters in nuclear ring show an azimuthal age gradient only when the SFR is small, such that younger clusters tend to locate closer to the contact points between the ring and dust lanes.
\end{abstract}

Nuclear rings in barred-spiral galaxies are sites of intense star formation (e.g., Mazzuca et al. 2008 , 2011). Observations indicate that ring star formation exhibits multiple burst activities over a timescale a few Gyrs (e.g., Allard et al. 2006; Sarzi et al. 2007; van der Laan et al. 2013). Yet, what makes it last such a long period of time is unknown. To understand what controls star formation in nuclear rings, we have run hydrodynamic simulations of barred galaxies by including star formation feedback in the form of momentum injection. Models with and without spiral arms were explored, separately, in Seo \& $\operatorname{Kim}(2013,2014)$. Here, we summarize the main numerical results: the reader is referred to Seo \& Kim $(2013,2014)$ for technical details as well as more quantitative results and discussion.

We initially consider a two-dimensional, infinitesimally-thin, isothermal, self-gravitating gaseous disk with sound speed of $10 \mathrm{~km} \mathrm{~s}^{-1}$. The disk has an exponential distribution of surface density. Its rotation curve resembles normal disk galaxies with a velocity of $\sim 200 \mathrm{~km} \mathrm{~s}^{-1}$ at the flat part. The bar potential is modeled by a Ferrers prolate with major and minor axes of $5 \mathrm{kpc}$ and $2 \mathrm{kpc}$, respectivley. The bar mass fraction is set to $30 \%$ of the spheroidal components (bulge plus bar) inside $10 \mathrm{kpc}$, while its pattern speed is fixed to $33 \mathrm{~km} \mathrm{~s}^{-1} \mathrm{kpc}^{-1}$. The spiral arms are represented by two-armed, trailing logarithmic spirals with a pitch angle of $20^{\circ}$. The arm strength is chosen such that the associated radial force is $10 \%$ of the centrifugal force arising from the background galaxy rotation (e.g., Kim et al. 2014). To study the effect of arm-induced gas inflows on the star formation rate (SFR) in a ring, we vary the arm pattern speed $\Omega_{\text {arm }}$ in the range of $\sim 10-33 \mathrm{~km} \mathrm{~s}^{-1} \mathrm{kpc}^{-1}$.

Figure 1 plots the temporal changes of (left) the SFR, (middle) the total stellar mass $M_{*}(t)$ formed until $t$, and (right) the total gas mass $M_{\text {ring }}$ in the ring for the bar-only model (dashed lines) and the models with spiral arms of differing $\Omega_{\mathrm{arm}}$ (solid lines). The SFR history of the bar-only model is characterized by a strong primary burst at $t \sim 0.1-0.2$ Gyr when the gas in the bar region experiences massive inflows due to the bar growth. There is no significant change in $M_{*}(t)$ after $t=0.3 \mathrm{Gyr}$ since the gas inflow rate to the ring becomes very small. Note that the presence of spiral arms makes the SFR rejuvenated at $t>0.4 \mathrm{Gyr}$, only when the arm pattern speed is smaller than that of the bar. This is because only the gas located between the bar ends and the corotation radius of the arms can move inward to the bar region by losing angular momentum, while the gas outside the corotation radius of the arms moves radially outward. Since the mass inflows due to the arms to the ring occur intermittently, temporal variations of the SFR at late time show episodic bursts. The associated mass inflow is larger for smaller 

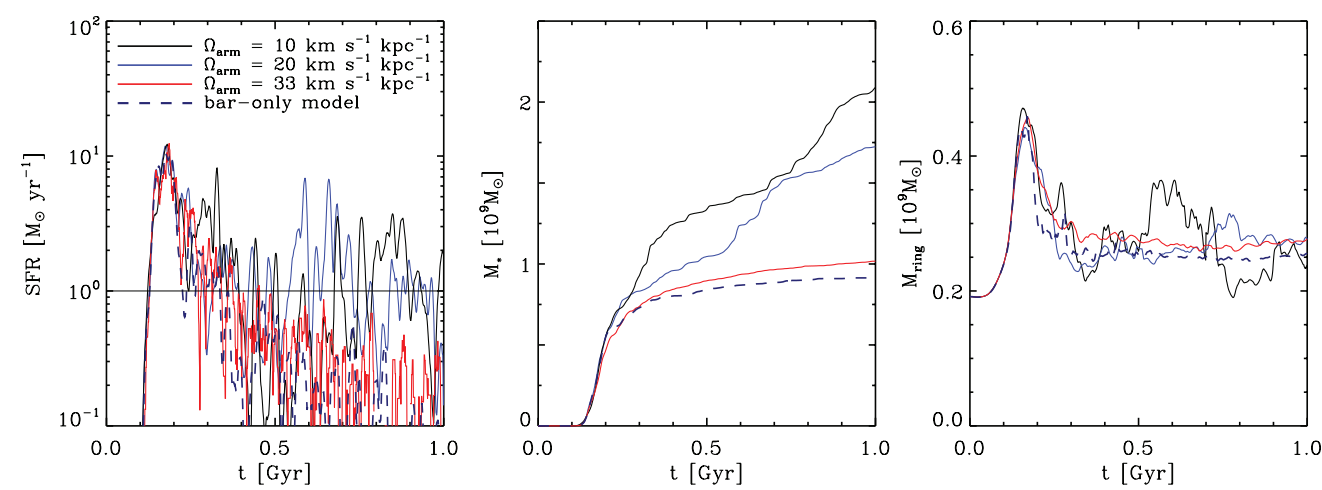

Figure 1. Temporal variations of the (left) SFR in the ring, (middle) total stellar mass $M_{*}(t)$ formed until $t$, and (right) total gas mass $M_{\text {ring }}$ in the ring for models with varying $\Omega_{\text {arm }}$. The results of the bar-only model are compared as dashed lines. Arm-induced gas inflows make the SFR rejuvenated at $t>0.4$ Gyr. The ring SFR is not well correlated with $M_{\text {ring }}$.

$\Omega_{\text {arm }}$ (Kim \& Kim 2014), resulting in larger $M_{*}$ at 1 Gyr. Note also that $M_{\text {ring }}$ in all models is maintained relatively constant at $\sim 2 \times 10^{8} M_{\odot}$, suggesting that it is not the gas mass in the ring but the mass inflow rate to the ring that controls the ring SFR.

We also found that when the SFR is large (larger than $1 M_{\odot} \mathrm{yr}^{-1}$ for our adopted model parameters), star formation is widely distributed throughout the ring, with no apparent age gradient. When the SFR is small, on the other hand, ages of young star clusters exhibit an azimuthal gradient along the ring, such that younger clusters are located closer to the contact points between the ring and dust lanes, since star formation takes place preferentially near the contact points. This is consistent with the observational result of van der Laan et al. (2013) that the ring star formation in NGC 6951 occurs in a "pearls-on-a-string" fashion to exhibit an age gradient in the azimuthal direction, with the mean SFR of $\sim 0.6 M_{\odot} \mathrm{yr}^{-1}$ during the past 1 Gyr.

\section{References}

Allard, E. L., Knapen, J. H., Peletier, R. F., \& Sarzi, M. 2006, MNRAS, 371, 1087

Kim, W.-T., Kim, Y., \& Kim, J.-G. ApJ, 789, 68

Kim, Y., \& Kim, W.-T. 2014, MNRAS, 440, 208

Mazzuca, L. M., Knapen, J. H., Veilleux, S., \& Regan, M. W. 2008, ApJ, 174, 337

Mazzuca, L. M., Swaters, R. A., Knapen, J. H., \& Veilleux, S. 2011, ApJ, 739, 104

Sarzi, M., Allard, E. L., Knapen, J. H., \& Mazzuca, L. M. 2007, MNRAS, 380, 949

Seo, W.-Y., \& Kim, W.-T. 2013, ApJ, 769, 100

Seo, W.-Y., \& Kim, W.-T. 2013, ApJ, 792, 47

van der Laan, T. P. R., Schinnerer, E., Emsellem, E., et al. 2013, A\& A, 551, A81 